\title{
PAR4 overexpression promotes colorectal cancer cell proliferation and migration
}

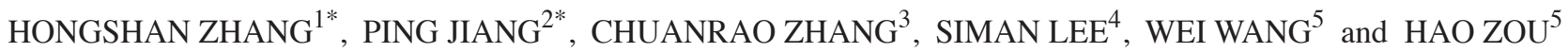 \\ ${ }^{1}$ Department of Cardiac Function, The Second Affiliated Hospital of Kunming Medical University, \\ Kunming, Yunnan 650101; Departments of ${ }^{2}$ Pathology and Pathophysiology, ${ }^{3}$ Functional Experimental Center and \\ ${ }^{4}$ Biochemistry, Kunming Medical University, Kunming, Yunnan 650500; ${ }^{5}$ Department of Hepatopancreatobiliary \\ Surgery, The Second Affiliated Hospital of Kunming Medical University, Kunming, Yunnan 650101, P.R. China
}

Received February 15, 2017; Accepted August 2, 2018

DOI: $10.3892 / 01.2018 .9407$

\begin{abstract}
Protease-activated receptor 4 (PAR4), a member of the G-protein-coupled receptor family, was previously identified to be involved in the progression of cancer. Previous study revealed that the expression of PAR4 was increased in colorectal cancer tissues compared with the associated normal tissues, particularly in positive lymph node and poorly differentiated types of cancer. We hypothesized that PAR4 serves a function in the progression of colorectal cancer. In the present study, overexpression of PAR4 in colorectal cancer LoVo cells promoted proliferation, anchorage-independent growth and migration. In vivo, PAR4 increased LoVo cell tumorgenicity. In contrast, knockdown of PAR4 in HT-29 cells decreased proliferation, anchorage-independent growth and migration. Mechanistic studies revealed that PAR4 increased the phosphorylation of extracellular-signal-regulated kinase $1 / 2$ in colorectal cancer cells, which is the potential molecular mechanism that promotes cellular proliferation and migration. Taken together, the results of the present study indicated that overexpression of PAR4 promoted colorectal cancer cell proliferation, survival and metastasis, indicating that PAR4 is a promising therapeutic target for preventing colon cancer progression.
\end{abstract}

\section{Introduction}

Colorectal cancer (CRC) is the most prevalent type of cancer and the second most important cause of cancer-associated mortality in Western countries (1), and the incidence of CRC

Correspondence to: Dr Hao Zou, Department of Hepatopancreatobiliary Surgery, The Second Affiliated Hospital of Kunming Medical University, 374 Dianmian Road, Kunming, Yunnan 650101, P.R. China

E-mail: newzouhao@hotmail.com

*Contributed equally

Key words: protease-activated receptor 4, colorectal cancer, proliferation, migration is increasing in certain countries of Eastern Europe and Asia (2). The progression from normal colonic mucosa to malignant tumor is a multistep process, which is involved in many genetic alternations. Chromosomal instability, primarily including adenomatous polyposis coli (APC) gene mutation, and the inactivation of the mismatch repair gene system are the potential molecular mechanisms that lead to CRC $(3,4)$. Proteinase-activated receptors (PARs), a subfamily of G-protein-coupled receptors, composed of four members, PAR1, PAR2, PAR3 and PAR4 (5), have been identified to be involved in the progression of various types of cancer (6-10). PAR1 and PAR2 were widely expressed in adenocarcinomas, melanomas, osteosarcomas, glioblastomas, meningiomas, leukemias, and breast and colon cancer. PAR1 and PAR2 promote cancer cell proliferation, motility and metastasis (11-13). PAR3 was expressed in kidney and liver cancer $(14,15)$. In human pancreatic adenocarcinoma PANC-1 cells, knockdown of PAR3 markedly enhanced cell migration and invasion (16). PAR4 was also expressed in multiple tumors. In prostate cancer, PAR4 expression was increased compared with the normal glands; however, no correlation between PAR4 expression and Gleason score was identified (17,18). PAR4 mRNA was identified in 10/14 (71\%) human colon cancer cell lines. PAR4 protein expression was absent from normal colon mucosa, but appeared as evident staining in the dysplastic and colon cancerous mucosa (19). In our previous study, we identified that PAR4 expression was increased in colon cancer compared with the associated normal tissue, and the upregulated expression was associated with lymph node invasion and cell differentiation (6). However, the effect of PAR4 on CRC cell proliferation, survival and migration, and the potential molecular mechanism involved in the functions have, to the best of our knowledge, not been investigated. We hypothesized that PAR4 promoted the proliferation and migration of CRC cells. Therefore, PAR4 was overexpressed in CRC LoVo cells or knocked down in HT-29 cells, and the effect of PAR4 on the proliferation and migration of CRC cells, and the phosphorylation level of extracellular-signal-regulated kinase (ERK)1/2 were investigated. The results of the present study indicated that overexpression of PAR4 promoted proliferation, survival and migration of CRC cells, and increased the phosphorylation 
level of ERK1/2. These observations suggested that PAR4 is involved in the progression of CRC.

\section{Materials and methods}

Ethics statement. The present study was reviewed and approved by The Second Affiliated Hospital of Kunming Medical University. All animals were raised according to the protocols approved by the Kunming Medical Experimental Animal Care Commission.

RNA extraction and quantitative polymerase chain reaction $(q P C R)$. RNA extraction, first-strand cDNA synthesis and the design of primers were as described previously (7). The primers used for qPCR were as follows: GAPDH (107 bp product; internal control), 5'-TGATGACATCAAGAAGGT GGTGAAG-3' (forward) and 5'-TCCTTGGAGGCCATG TGGGCCAT-3' (reverse); PAR4 (147 bp product), 5'-CCTTCA TCTACTACTACTACGTGTCG-3' (forward) and 5'-ACTGGA GCAAAGAGGAGTGG-3' (reverse). qPCR for PAR4 and GAPDH were performed using an SYBR Green Real-Time PCR kit (Thermo Fisher Scientific, Inc., Waltham, MA, USA) with the conditions as follows: Initial denaturation at $95^{\circ} \mathrm{C}$ for $1 \mathrm{~min}$, followed by 40 cycles of $95^{\circ} \mathrm{C}$ for $15 \mathrm{sec}$ and $60^{\circ} \mathrm{C}$ for $1 \mathrm{~min}$. Each sample was run three times. Products were analyzed using a continuous fluorescence detector with Opticon Monitor 3.0 software (Bio-Rad Laboratories, Inc., Hercules, CA, USA). No-template controls (no cDNA in PCR) were included to detect non-specific or genomic amplification and primer dimerization. Relative quantitative evaluation of PAR4 was performed using the E-method $(6,7)$ and expressed as a ratio of the transcript of PAR4 to GAPDH in the different cell lines. The identities of qPCR products were confirmed by DNA sequencing.

Western blot analysis. Mouse anti-human PAR4 monoclonal antibody (cat. no. sc-130078; 1:1,000) and mouse anti-human $\beta$-actin monoclonal antibody (cat. no. sc-517582; 1:1,000) were from Santa Cruz Biotechnology, Inc. (Dallas, TX, USA), and mouse anti-human phospho-ERK1/2 monoclonal antibody (cat. no. 9106; 1:2,000) and rabbit anti-human ERK1/2 polyclonal antibody (cat. no. 9102; 1:1,000) were from Cell Signaling Technology, Inc. (Danvers, MA, USA). Cells $\left(1 \times 10^{4}\right)$ were washed twice with ice-cold PBS and were lysed with radioimmunoprecipitation lysis buffer (Thermo Fisher Scientific, Inc.) containing a protease and phosphatase inhibitor cocktail (Roche Diagnostics, Basel, Switzerland). The protein concentration was determined using a Quick Start ${ }^{\mathrm{TM}}$ Bradford protein assay kit (cat. no. 5000201; Bio-Rad Laboratories, Inc.). Samples (20 $\mu \mathrm{g}$ protein) were separated by SDS-PAGE (30\% acrylamide) and then transferred onto a polyvinyl difluoride membrane. The membrane was subsequently blocked with $3 \%$ bovine serum albumin diluted in TBS with $0.1 \%$ Tween-20 (1:1,000; cat. no. T8220; Beijing Solarbio Science and Technology Co., Ltd., Beijing, China) and incubated with the primary antibody and the horseradish peroxidase-labeled goat anti-rabbit (cat. no. SE134; 1:1,000) and anti-mouse (cat. no. SE131; 1:1,000) (both from Beijing Solarbio Science and Technology Co., Ltd.). Proteins were visualized using Super Signal reagents (Pierce; Thermo Fisher Scientific, Inc.) with the ChemiDoc XRS imaging system (Bio-Rad Laboratories, Inc.) and analyzed using the Image Lab 5.2 software (Bio-Rad Laboratories, Inc.).

Cell culture. Human CRC LoVo and HT-29 cell lines were from the American Type Culture Collection (Manassas, VA, USA). LoVo cells were cultured in Ham's F-12K (Kaighn's) medium (cat. no. 21127022; Pierce; Thermo Fisher Scientific, Inc.) supplemented with $10 \%(\mathrm{v} / \mathrm{v})$ fetal bovine serum (FBS; Gibco; Thermo Fisher Scientific, Inc.), $100 \mathrm{U} / \mathrm{ml}$ penicillin and 100 U/ml streptomycin (Gibco; Thermo Fisher Scientific, Inc.), and HT-29 cells were cultured in Dulbecco's modified Eagle's medium (Gibco; Thermo Fisher Scientific, Inc.) supplemented with $10 \%$ (v/v) FBS, $100 \mathrm{U} / \mathrm{ml}$ penicillin and $100 \mathrm{U} / \mathrm{ml}$ streptomycin. Cells were cultured at $37^{\circ} \mathrm{C}$ in a humidified atmosphere containing $5 \% \mathrm{CO}_{2}$.

Generation of LoVo cells overexpressing PAR4. cDNA encoding human PAR4 was inserted into bicistronic retroviral vector to obtain pBMN-PAR4-NEO. pBMN-PAR4-NEO was used to produce retrovirus containing PAR4, and LoVo cells were infected using the retrovirus. In brief, retrovirus expressing PAR4 was generated by transfecting pBMN-PAR4-NEO plasmid into Phoenix cells (Pierce; Thermo Fisher Scientific, Inc.). At $48 \mathrm{~h}$ after transfection, the retrovirus supernatant was collected, centrifuged $(1,000 \mathrm{x}$ g for $3 \mathrm{~min}$ at room temperature) and filtered through a $0.45 \mu \mathrm{m}$ filter. LoVo/PAR4 cells were produced using the retrovirus supernatant with PAR4, and $\mathrm{LoVo} /$ vector cells were generated using the retrovirus supernatant with pBMN-I-NEO only. G418 $(600 \mu \mathrm{g} / \mathrm{ml})$ was used to select the stable overexpression of PAR4 (LoVo/PAR4) and the empty vector (LoVo/vector).

Knockdown of PAR4 in HT-29 cells. Since PAR4 was expressed in CRC HT-29 cells, short hairpin RNA (shRNA) was used to knock down PAR4 in HT-29 cells. shRNA targeting PAR4 was from GE Healthcare Dharmacon, Inc. (Lafayette, CO, USA). Briefly, lentivirus expressing PAR4 shRNA was generated by co-transfecting PAR4 shRNA plasmids with pCMV-dR8.2 dvpr and pCMV-VSVG packaging plasmids into 293FT cells (Shanghai Genechem Co., Ltd., Shanghai, China.). At 48 h after transfection, the lentiviral supernatant was collected, centrifuged $(1,000 \mathrm{x}$ g for $3 \mathrm{~min}$ at room temperature) and filtered through a $0.45 \mu \mathrm{m}$ filter. HT-29 cells were infected with collecting lentivirus supernatant with shPAR4. The lentiviral vector pGIPZ, containing puromycin and green fluorescent protein (GFP) selection markers, was used to knock down PAR4. HT-29/pGIPZ cells were generated using the lentivirus supernatant with pGIPZ only as control. HT-29 cells transduced with pGIPZ only or pGIPZ-shPAR4 were selected using $5 \mu \mathrm{g} / \mathrm{ml}$ puromycin to generate HT-29/pGIPZ or HT-29/shPAR4, respectively.

Cell proliferation assay. Cell proliferation was determined by viable cell counting in Dulbecco's modified Eagle's (high-glucose) medium (Gibco; Thermo Fisher Scientific, Inc.) containing $10 \% \mathrm{FBS}$. LoVo/vector or LoVo/PAR4 cells were seeded in a 6 -well plate with each containing $1 \times 10^{5}$ cells in $3 \mathrm{ml}$ culture medium individually The number of cells was determined daily for 5 days using a 
A

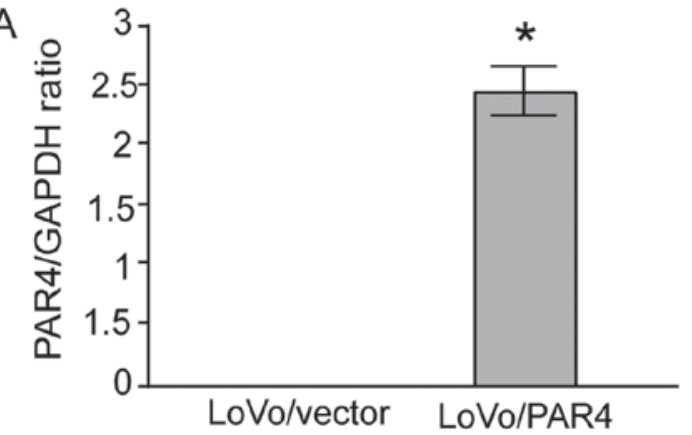

C

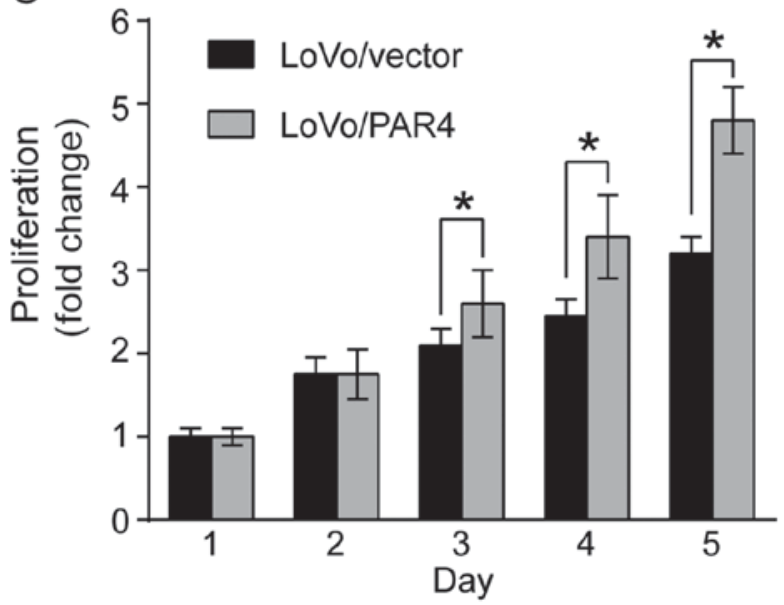

B

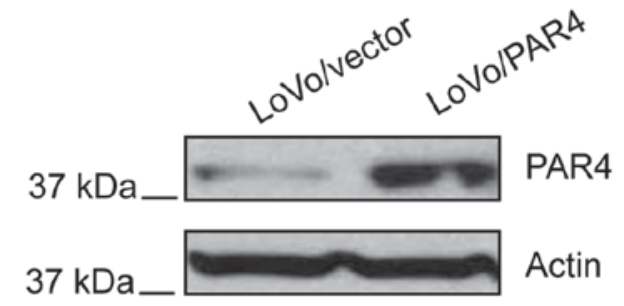

D

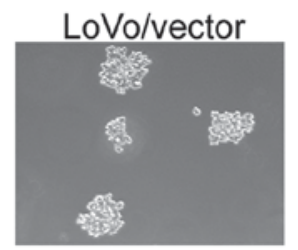

LoVo/PAR4

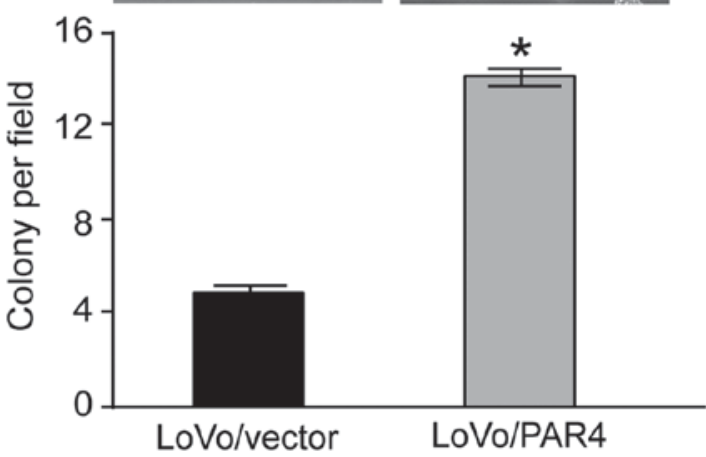

Figure 1. PAR4 increases LoVo cell proliferation and survival. (A) Quantitative polymerase chain reaction analysis of PAR4 from LoVo/vector and LoVo/PAR4 cells. " $\mathrm{P}<0.05$ vs. LoVo/vector cells. (B) Western blot analysis of PAR4 of whole cell lysates from LoVo/vector and LoVo/PAR4 cells. $\beta$-actin was used as a loading control. (C) Proliferation of LoVo/vector and LoVo/PAR4 cells in the presence of $10 \%$ fetal bovine serum. Results are expressed as the fold change in cell number relative to day $1(\mathrm{n}=3)$. ${ }^{*} \mathrm{P}<0.05$. (D) Number of colonies observed from LoVo/vector and LoVo/PAR4 cells in soft agar containing $5 \%$ fetal bovine serum. ${ }^{*} \mathrm{P}<0.05$ vs. LoVo/vector cells. PAR4, protease-activated receptor 4.

hemocytometer and the fold increase in cell proliferation was calculated.

Cell survival assay. The soft agar colony assay was performed as described by $\mathrm{Yu}$ et al (20). In brief, $2 \times 10^{4} \mathrm{LoVo} /$ vector or LoVo/PAR4 cells were seeded in the $0.35 \%$ top agarose in culture medium with $5 \%$ FBS, and the base agarose concentration was $0.7 \%$ in the same medium in a 6-well plate, and the cells were fed every 3 days with culture medium containing $5 \%$ FBS. The colony number was quantified in 10 randomly selected visual fields when the cells were cultured for 2 weeks.

In vivotumorigenicityassay. The animal protocol was designed to minimize pain or discomfort to the animals. The female BALB/c nude mice with 4-6 weeks old were obtained from Experimental Animal Center of Kunming Medical University (Kunming, China), and were randomly divided into 2 groups ( $n=4 /$ group). The animals (weight, $21.19 \pm 1.45 \mathrm{~g}$ ) were acclimatized to laboratory conditions $\left(23^{\circ} \mathrm{C}, 12\right.$-h light/12-h dark cycle, $50 \%$ humidity, ad libitum access to food and water) for 2 weeks prior to experimentation. A total of $1 \times 10^{6} \mathrm{LoVo} / \mathrm{PAR} 4, \mathrm{LoVo} /$ vector cells, HT-29/pGIPZ, HT-29/shPAR4\#4 or HT-29/shPAR4\#5 cells in $50 \mu \mathrm{l}$ PBS were injected subcutaneously into the two sides of the male nude mice. Tumor growth was monitored weekly by determining the tumor volume using the formula $\mathrm{V}=\left(\mathrm{W}^{2} \times \mathrm{L}\right) / 2$, where $\mathrm{V}$ is volume; $\mathrm{W}$ is width and $\mathrm{L}$ is length. After 5 weeks, the mice were sacrificed, and the tumors were isolated and weighed. Animals were sacrificed immediately on presentation of signs of pain, distress, suffering or impending mortality.

Cell migration assay. Migratory activities of LoVo/PAR4, LoVo/vector cells, HT-29/pGIPZ, HT-29/shPAR4\#4 or HT-29/shPAR4\#5 cells in vitro were determined using a Matrigel insert migration assay. Following starvation for $24 \mathrm{~h}$, $3 \times 10^{5}$ cells in $300 \mathrm{ml}$ serum-free medium were seeded into a FluoroBlok ${ }^{\mathrm{TM}}$ Cell Culture insert (Corning Incorporated, Corning, NY, USA). The lower chamber of a 24-well plate contained $500 \mathrm{ml}$ pre-warmed culture medium containing $10 \%$ FBS. At $16 \mathrm{~h}$ after seeding, the non-migrating cells remaining in the insert were scraped off using a cotton swab and the migrated cells in the bottom part of the insert were labeled with calcein acetoxymethyl ester (Invitrogen; Thermo Fisher Scientific, Inc.) in culture medium containing $10 \%$ FBS. Cells that had migrated through the membranes were quantified by determining the number of cells in five randomly selected visual fields.

Statistical analysis. Results are expressed as the mean \pm standard deviation. Differences between the groups were evaluated using a paired Student's t-test or one-way analysis of variance. Statistical analyses were performed using SPSS software for Windows (version 21.0; IBM Corp., 
A

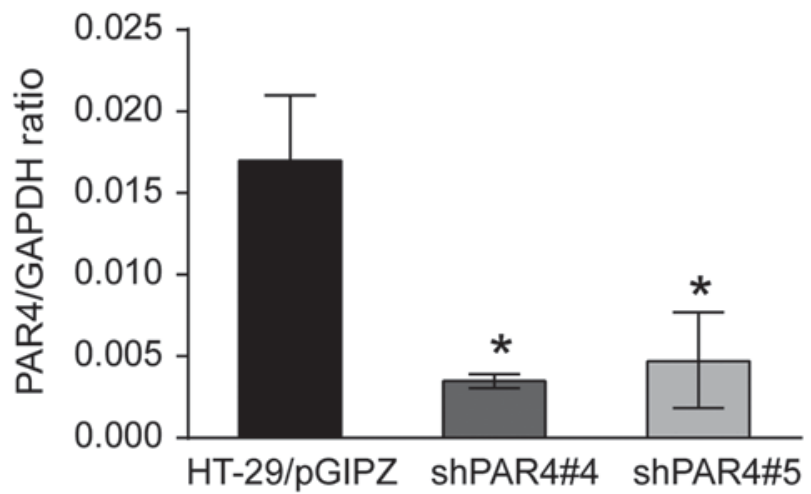

C

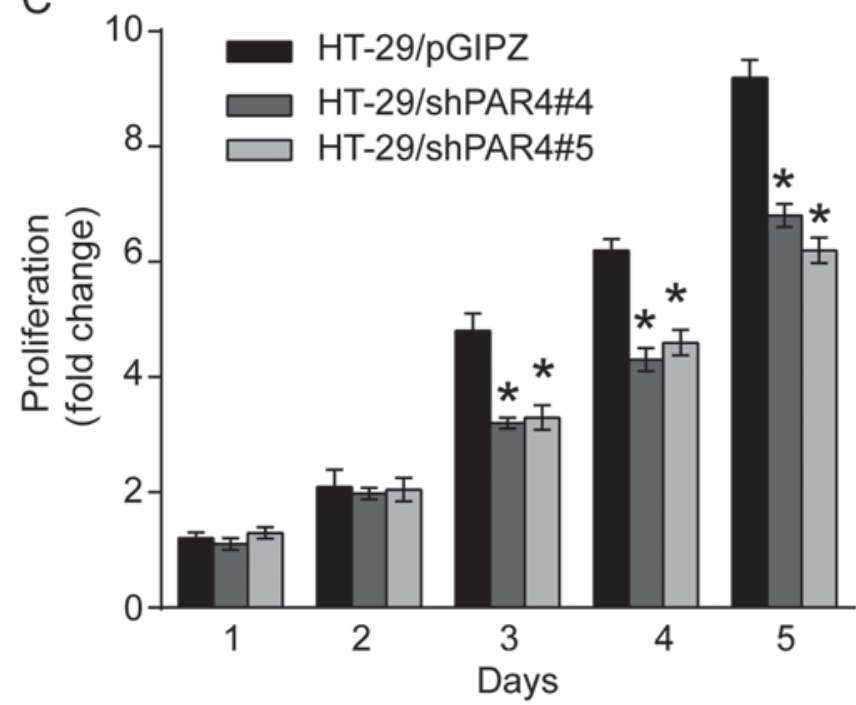

B

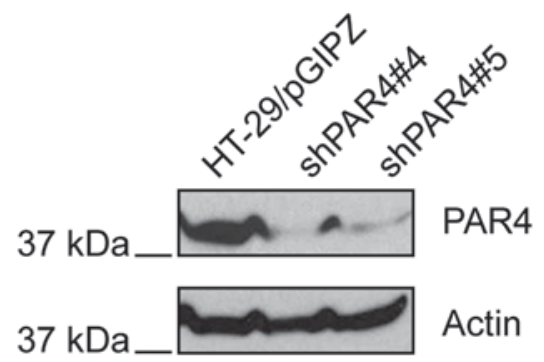

D
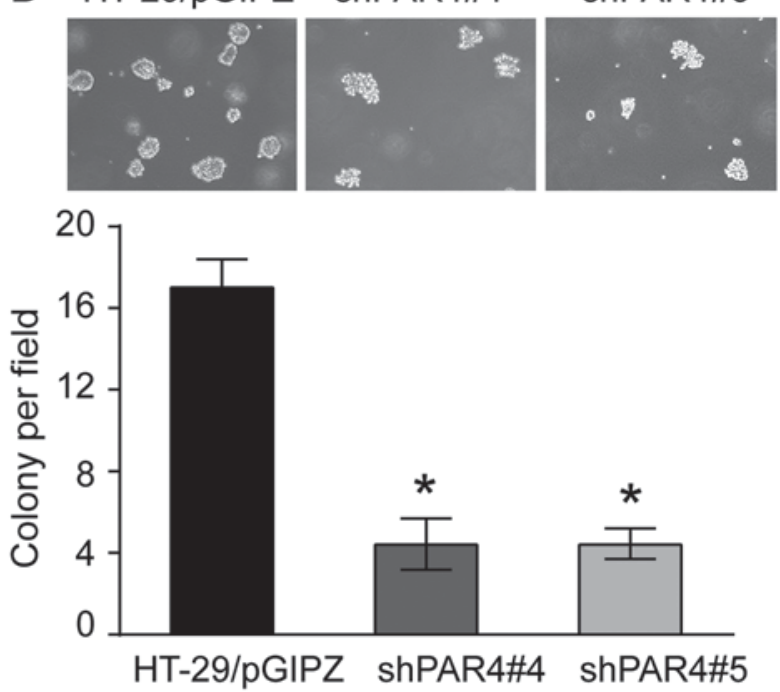

Figure 2. PAR4 is required for HT-29 cell proliferation and survival. (A) Quantitative polymerase chain reaction analysis of PAR4 expression from HT-29 cells transfected with empty vector (pGIPZ) or shPAR4 (clones \#4 and \#5). (B) Western blot analysis of PAR4 of whole cell lysates from HT-29/shPAR4\#4 and HT-29/shPAR4\#5 cells. $\beta$-actin was used as a loading control. (C) Proliferation of HT-29/pGIPZ, HT-29/shPAR4\#4 or shPAR4\#5 cells in the presence of $10 \%$ fetal bovine serum. Results are expressed as the fold change in cell number relative to day 1 (n=3). (D) Number of colonies observed from HT-29/pGIPZ, HT-29/shPAR4\#4 or shPAR4\#5 cells in soft agar containing 5\% FBS. * P<0.05 vs. HT-29/pGIPZ cells. PAR4, protease-activated receptor 4; shPAR4, short hairpin RNA targeting PAR4.

Armonk, NY, USA). $\mathrm{P}<0.05$ was considered to indicate a statistically significant difference.

\section{Results}

PAR4 increases LoVo cell proliferation and survival. To investigate the effect of PAR4 on CRC cell proliferation, PAR4 was overexpressed in LoVo cells. qPCR analysis indicated that mRNA expression of PAR4 was increased significantly in LoVo/PAR4 cells compared with in LoVo/vector cells (Fig. 1A). Western blot analysis revealed that PAR4 protein was increased in LoVo/PAR4 cells compared with in the LoVo/vector cells (Fig. 1B). The effect of overexpression of PAR4 on LoVo cells was investigated. In culture medium containing $10 \% \mathrm{FBS}$, from day 3 onwards, a significant difference in cell proliferation activity between LoVo/PAR4 cells and the control LoVo/vector cells was identified (Fig. 1C), indicating that PAR4 promotes LoVo cell proliferation under standard culture conditions. It was next investigated whether the PAR4-mediated proliferation leads to increased anchorage-independent growth in soft agar. When LoVo/vector cells were plated in soft agar, none or few colonies were observed when the colonies were cultured for 2 weeks, whereas a significant number of LoVo/PAR4 cells colonies was observed. Quantification indicated that expression of PAR4 led to a $>3$-fold increase in colony formation of LoVo cells (Fig. 1D). Taken together, these results indicated that increased expression of PAR4 enhances proliferation and survival of LoVo cells.

Knockdown of PAR4 decreases proliferation and anchorage-independent growth of HT-29 cells. Since overexpression of PAR4 promotes LoVo cell proliferation, the effect of proliferation of knocking down PAR4 on CRC cells as investigated. Since PAR4 was expressed in CRC HT-29 cells (21), lentiviral shRNA was used to knock down PAR4 in HT-29 cells. The lentiviral vector pGIPZ, containing puromycin and GFP selection markers, was used to knock down PAR4. HT-29 cells with knockdown of PAR4 were generated with $5 \mu \mathrm{g} / \mathrm{ml}$ puromycin resistance selection. HT-29 cells transfected with pGIPZ vector were selected using $5 \mu \mathrm{g} / \mathrm{ml}$ puromycin and were used as a control. qPCR indicated that PAR4 mRNA expression was decreased in HT-29/shPAR4\#4 $(\mathrm{P}<0.05)$ or HT-29/shPAR4\#5 $(\mathrm{P}<0.05)$ clones compared with HT-29/pGIPZ control cells (Fig. 2A). Western blotting using PAR4-specific antibody 
A

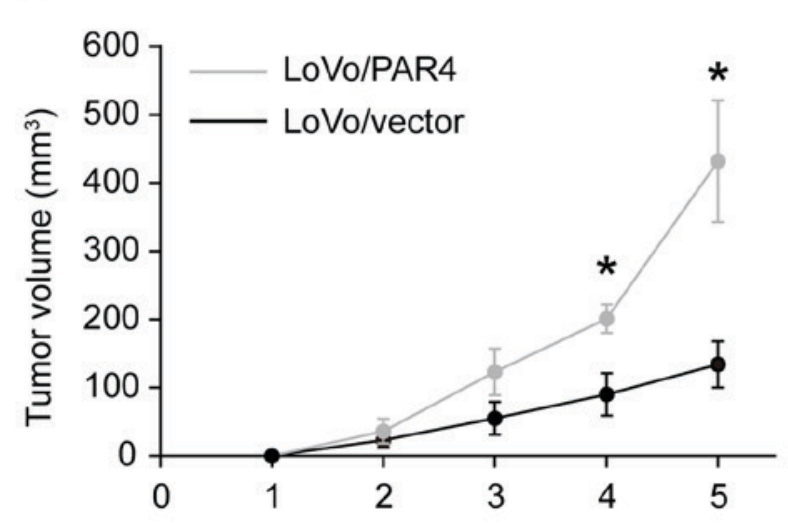

B
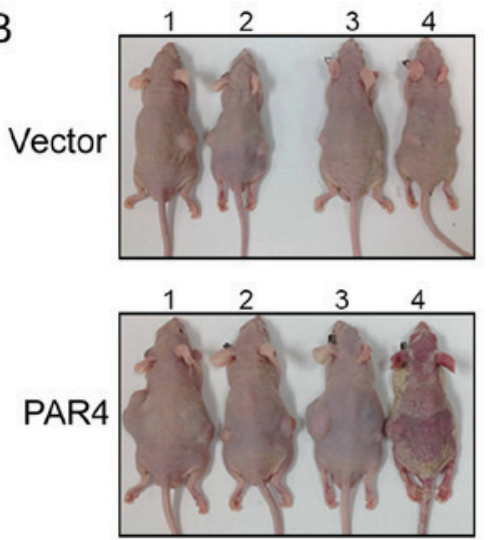
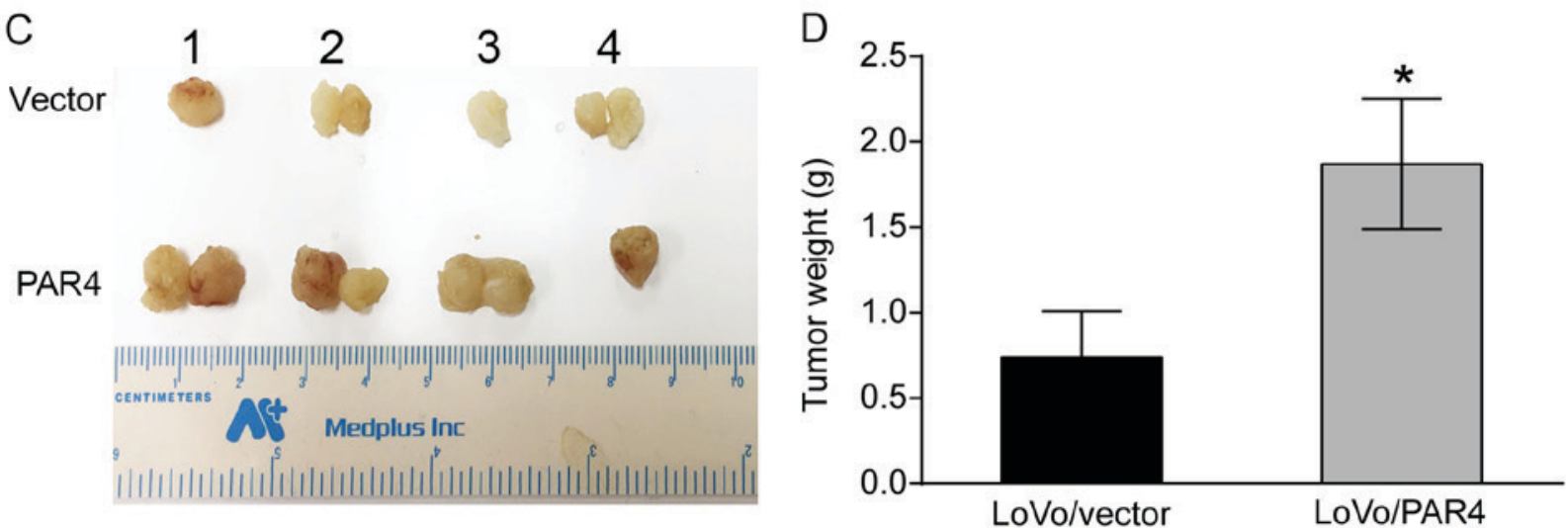

Figure 3. Overexpression of PAR4 in LoVo cells increases LoVo tumorigenesis. (A) LoVo/vector or LoVo/PAR4 cells were separately injected into nude mice subcutaneously, and the tumor volume was quantified weekly. The LoVo/PAR4 tumor increased in volume more rapidly compared with the LoVo/vector tumor, and a significant difference in tumor volume between the LoVo/vector and LoVo/PAR4 was observed from week 3 onwards. (B) Mice injected with LoVo/vector and LoVo/PAR4 cells, and (C) tumors from LoVo/vector and LoVo/PAR4 mice. (D) Average tumor weights from mice 5 weeks after injection with $\mathrm{LoVo} /$ vector and LoVo/PAR4 cells. The tumor volumes and weights are expressed as the mean \pm standard deviation. "P $<0.05$ vs. LoVo/vector. PAR4, protease-activated receptor 4.

was used to confirm the knockdown of PAR4 in these cell lines. As expected, the protein expression level of PAR4 in HT-29/shPAR4\#4 and HT-29/shPAR4\#5 cells was markedly decreased compared with HT-29/pGIPZ control cells (Fig. 2B).

The effect of knockdown of PAR4 on HT-29 cell proliferation was investigated. As presented in Fig. 2C, the proliferative activities of HT-29/shPAR4\#4 and HT-29/shPAR4\#5 clones were significantly decreased compared with HT-29/pGIPZ control in standard culture medium containing 10\% FBS. Similarly, in the colony formation assay, when these cells were plated in agarose with culture medium containing 5\% FBS and cultured for 2 weeks, colony formation of HT-29/shPAR4\#4 or HT-29/shPAR4\#5 cells in soft agar was decreased significantly compared with HT-29/pGIPZ cells after 2 days. Determination of the colony numbers indicated that knockdown of PAR4 in HT-29 cells led to a 4-fold decrease in colony formation compared with the HT-29/pGIPZ control cells $(\mathrm{P}<0.05$; Fig. 2D). The results suggested that knockdown of PAR4 in HT-29 cells significantly decreased the proliferation and survival of CRC HT-29 cells.

Overexpression of PAR4 increases LoVo cell tumorigenesis. Since PAR4 overexpression increased LoVo cell proliferation and survival, the effect of PAR4 on tumorigenesis was investigated. LoVo/vector and LoVo/PAR4 cells were injected into the two sides of each nude mouse subcutaneously, and the tumor volume was determined weekly. As presented in Fig. 3A, quantification of the tumor volume over 5 weeks indicated that the LoVo/PAR4 tumor volume increased more rapidly compared with the $\mathrm{LoVo} /$ vector tumor volume, and there was as significant difference between LoVo/PAR4 and LoVo/vector from 3 weeks onwards. When the mice were sacrificed in week 5 , it was identified that there were six tumors present in the LoVo/vector group, but seven tumors present in the LoVo/PAR4 group. The tumor of mouse no. 1 in PAR4/LoVo group exhibited the longest diameter $(1.83 \mathrm{~cm})$. Multiple tumors was observed for mouse no. 2 (two tumors; 0.3 and $0.5 \mathrm{~cm}$ ), no. 3 (two tumors; 0.2 and $0.5 \mathrm{~cm}$ ) and no. 4 (two tumors; 0.2 and $0.3 \mathrm{~cm}$ ) in the control group, and no. 1 (two tumors; 1.8 and $0.5 \mathrm{~cm}$ ), no. 2 (two tumors; 0.2 and $0.7 \mathrm{~cm}$ ) and no. 3 (two tumors; 1.7 and $0.2 \mathrm{~cm}$ ) in the LoVo/PAR4 group (Fig. 3B). The average tumor weight for the $\mathrm{LoVo} /$ vector group was $0.675 \pm 0.12 \mathrm{~g}$, and for the LoVo/PAR4 group was $1.875 \pm 0.09 \mathrm{~g}$, which suggested that there was significant difference between the tumor weight between LoVo/vector and LoVo/PAR4 group (Fig. 3C and D). The weights of the animals at the time of sacrifice were $1.87,1.89$, 


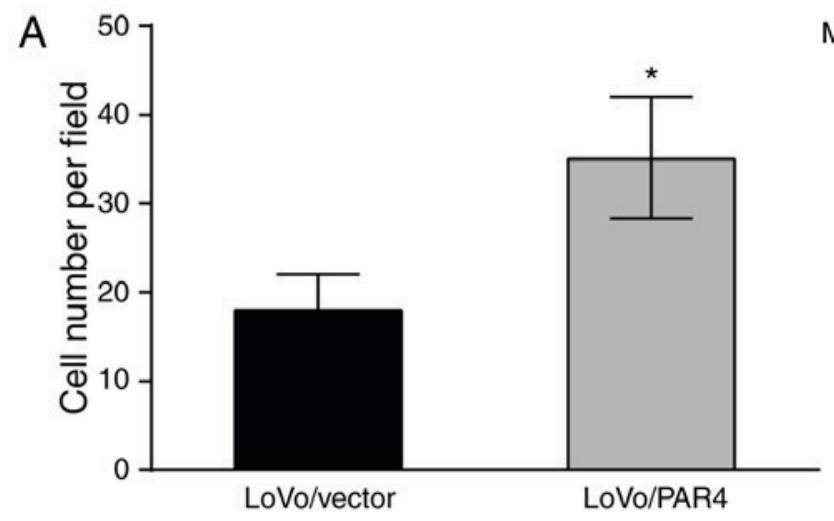

Migration
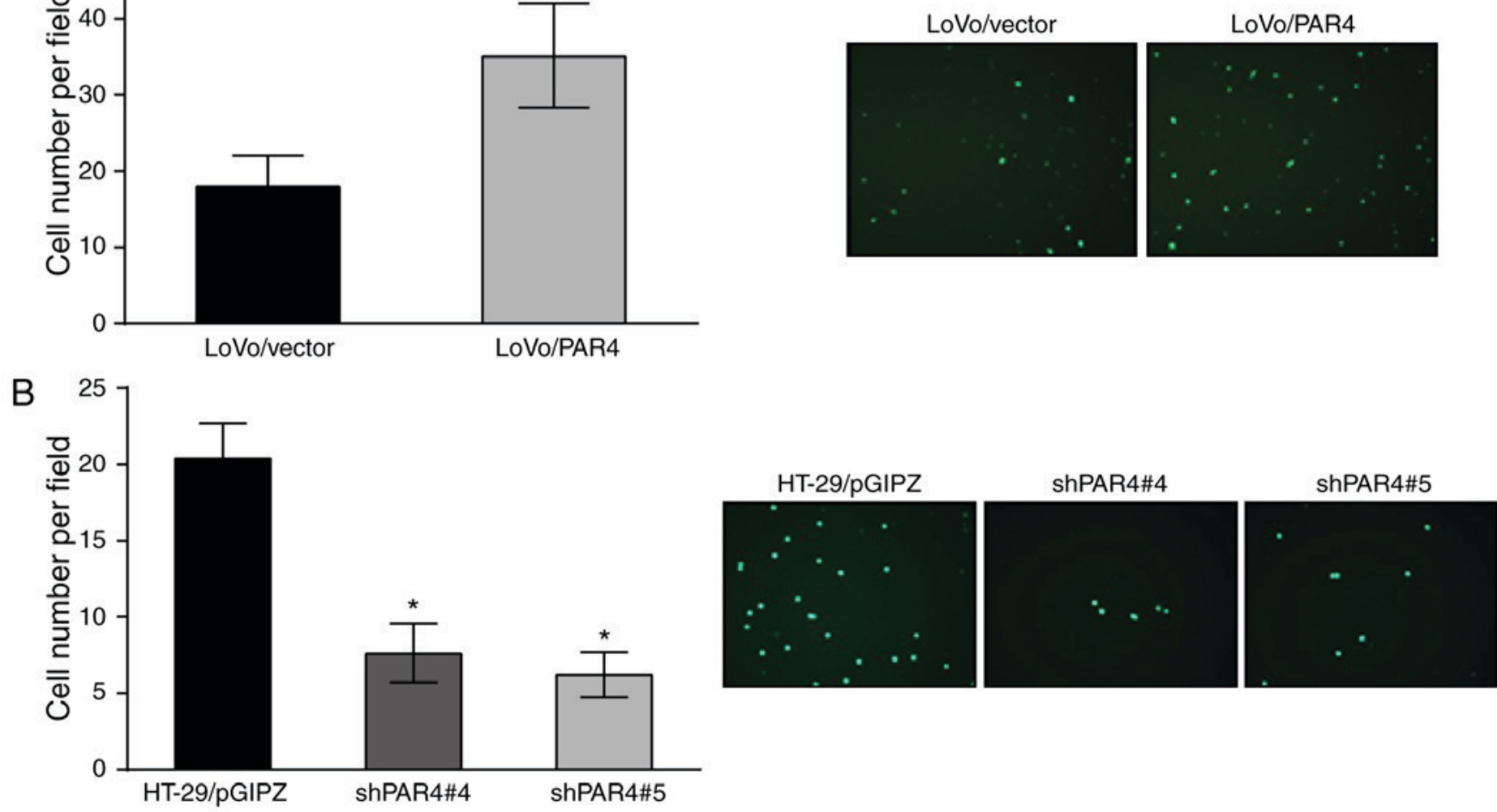

Figure 4. Effects of PAR4 on the migratory activity of colon cancer cells. (A) Migratory activity of LoVo/vector and LoVo/PAR4 cells. Left: Quantification of cells that migrated to the other side of the migration insert. Right: Migratory cells from an area randomly selected from five fields. "P<0.05 vs. LoVo/vector. (B) Migratory activity of HT-29/pGIPZ, HT-29/shPAR4\#4 and HT-29/shPAR4\#5cells. Left: Quantification of cells that migrated to the other side of the migration insert. Right: Migratory cells from an area randomly selected from five fields. "P<0.05 vs. HT-29/pGIPZ. PAR4, protease-activated receptor 4.

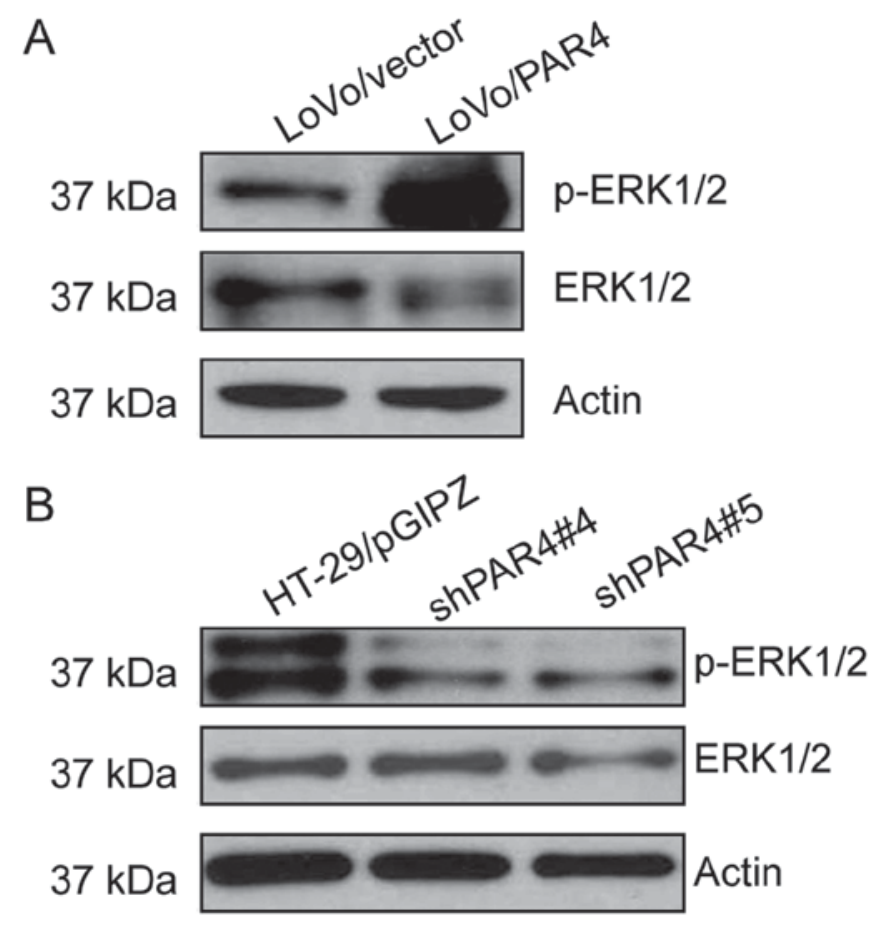

Figure 5. Overexpression of PAR4 activates ERK1/2. (A) Western blot analysis of phospho-ERK1/2 and total ERK1/2 of whole cell lysates from LoVo/vector and LoVo/PAR4 cells. $\beta$-actin was used as a loading control. (B) Western blot analysis of phospho-ERK1/2 and total ERK1/2 of whole cell lysates from HT-29/pGIPZ, HT-29/shPAR4\#4 and HT-29/shPAR4\#5cells. $\beta$-actin was used as a loading control. ERK, extracellular-signal-regulated kinase; p-, phospho-.
1.19 and $1.96 \mathrm{~g}$ for the LoVo/PAR4 group. The tumor burden of mouse no. 1 and no. 3 in the LoVo/PAR4 group was $>10 \%$ of its body weight, and the maximum tumor burden was $11.6 \%$. Furthermore, a 'cornmeal-like appearance' in mouse \#4 (but no other mouse) of the LoVo/PAR4 group may have been caused by an infection by Corynebacterium bovis, which healed after 6 days, and the mouse did not exhibit signs of pain or suffering during the experimental period. The alteration in the skin of this mouse had little effect on the results of the present study. The results indicated that expression of PAR4 promoted colorectal tumor growth, possibly through an increase in proliferation and survival of CRC cells. However, when HT-29/pGIPZ, HT-29/shPAR4\#4 or shPAR4\#5 cells were each injected into nude mice subcutaneously, there was no significant difference in tumor growth among them (data not shown).

Effects of PAR4 on the migration of CRC cells. To determine whether PAR4 is able to induce changes in colon cancer cell motility, the migration effect of PAR4 on LoVo cells and HT-29 cells was investigated. The migratory activity was investigated using a Transwell migration assay. As presented in Fig. 4A, LoVo/PAR4 cells exhibited increased migratory activity compared with LoVo/vector cells after $16 \mathrm{~h}$. Quantification of migratory cells indicated that expression of PAR4 led to a $>2$-fold increase compared with the LoVo/vector cells. Similarly, knockdown of PAR4 in HT-29 also decreased the migratory activity compared to the relative control cells. Quantification of migration of HT-29/shPAR4\#4 and HT-29/shPAR4\#5 cells demonstrated that knockdown of PAR4 $(\mathrm{P}<0.05)$ led to a $>2$-fold 
decrease in migratory activity compared with the HT-29/pGIPZ control (Fig. 4B). These results indicated that the expression of PAR4 increased colon cancer cell migration.

PAR4 activates ERK1/2 in CRC cells. In order to explore the potential underlying molecular mechanism for the effects of PAR4 in CRC cells, the expression level of phospho-ERK1/2 in CRC cells was investigated. As presented in Fig. 5, the level of phospho-ERK1/2 was increased in LoVo/PAR4 cells compared with the LoVo/vector cells. However, the level of phospho-ERK1/2 was decreased markedly in HT-29/shPAR4\#4 or HT-29/shPAR4\#5 clones compared with the HT-29/pGIPZ control (Fig. 5B). These results indicated that PAR4 overexpression in CRC cells increased the level of phospho-ERK1/2.

\section{Discussion}

PAR4, the most recently identified member of the PAR family, is a G-protein-coupled receptor, and serves physiological functions in the presence of thrombin, trypsin and cathepsin G (22). Besides platelet activation and relaxation of esophageal smooth muscle, PAR4 is involved in the progression of cancer $(23,24)$. In human hepatocellular carcinoma (HCC), PAR1 and PAR4 were identified to trigger $\mathrm{HCC}$ cell migration through activating common pro-migratory signaling pathways $(14,25)$. In gastric cancer, PAR1 and PAR2 promote gastric carcinogenesis by trigging intracellular signals, whereas PAR4 serves a negative function in the progression of gastric cancer (26). In our previous research, the expression of PAR4 was decreased in gastric, lung and esophageal cancer compared with the normal tissues, and the downregulation of expression was associated with poor cell differentiation and lymph node invasion $(7,9,10)$. However, the expression of PAR4 was increased in CRC tissues, and the upregulation of expression partly resulted from the hypomethylation of the promoter (6). In the present study, in order to investigate further the function of PAR4 in the progression of CRC, PAR4 was overexpressed in CRC LoVo cells, and it was identified that PAR4 promotes LoVo cell proliferation and anchorage-independent growth compared with the control. When PAR4 was knocked down in HT-29 cells, proliferation and colony formation of HT-29 cells were decreased compared with the vector only. The results suggested that PAR4 serves a function in the proliferation and survival of CRC cells. When the cells were injected into the nude mouse subcutaneously, it was identified that the tumor growth of LoVo/PAR4 was more rapid compared with that of the LoVo/vector, suggesting that PAR4 promoted colorectal tumorigenesis. The tumorigenesis of PAR4 is possibly a result of the effect of PAR4 increasing cell proliferation and survival. However, no significant difference in tumor growth was identified between HT-29/pGIPZ and HT-29/shPAR4\#4 or shPAR4\#5 cells (data not shown), which may be improved by increasing the number of animals in future research. In the present study, it was also identified that overexpression of PAR4 in LoVo cells promoted cell migration, and knockdown of PAR4 in HT-29 cells decreased cell migration. The results indicated that PAR4 promoted CRC cell migration. In fact, in Hep3B hepatocellular carcinoma cells, PAR4 and PAR1 have been identified to promote cell migration depending on reactive oxygen species formation and receptor tyrosine kinase transactivation (25). Similarly, the study of
Zhang et al (21) revealed that human trefoil factor 2 (hTFF2) promoted the invasion of gastric cancer cells that overexpressed PAR4, but there was no promotion effect when the PAR4 was knocked down. In our previous study, it was identified that the expression levels of PAR4 were markedly increased in CRC tissues compared with the matched non-cancerous tissues, particularly in cancer with positive lymph node metastasis (6). These results are consistent with the promotion effect on migration of PAR4, and suggested that PAR4 serves a function in lymph mode metastasis of CRC. Finally, the potential underlying molecular mechanism for the function of PAR4 in the progress of CRC was investigated. It was investigated that overexpression of PAR4 in LoVo cells increased the level of phospho-ERK1/2, and that knockdown of PAR4 in HT-29 cells decreased ERK1/2 expression. These results suggested that PAR4 increased the level of phospho-ERK1/2. In fact, it has been identified previously that the function of PAR4 was associated with the activation of the ERK2 signaling pathway. A study by Gratio et al (19) identified that the increase in the extracellular phosphorylation level of ERK1/2 and ErbB-2 was associated with the promotion effect of PAR4 on colon cancer cell proliferation. In recent research, Smith et al (27) identified that the internalization of activated PAR4 is associated with proper ERK1/2 and protein kinase B activation. Recombinant hTFF2 promoted gastrointestinal cancer AGS and LoVo cell migration via phosphorylation of ERK1/2 when PAR4 was overexpressed in the cells (21). In that study, it was identified that PAR4 activated the ERK1/2 signaling pathway, which is possibly involved in the promoted effect of CRC cell proliferation, migration and tumorigenesis.

In conclusion, the results of the present study indicated that PAR4 serves a function in proliferation and migration of colon cancer cells, which possibly results in tumorigenesis and invasion of CRC tumors. Further investigation of the molecular mechanism of the involvement of PAR4 in tumorigenesis and metastasis in CRC is warranted.

\section{Acknowledgements}

Not applicable.

\section{Funding}

This work was supported by the Chinese National Natural Science Foundation (grant nos. 81160302 and 81260084), and Yunnan Province Basic Research for Application Fund of Yunnan China (grant nos. 2012FB050 and 2011FZ124).

\section{Availability of data and materials}

The datasets used and/or analyzed during the current study are available from the corresponding author on reasonable request.

\section{Authors' contributions}

$\mathrm{HZ}$ and $\mathrm{WW}$ were involved in the design of the present study. HSZ, PJ, CZ and WW performed the cell experiments and interpreted the cell data. HSZ and PJ conducted the animal assays. HSZ and SL were involved in acquisition of data and performed the western blot analysis. SL and WW analyzed 
and interpreted the data. HSZ, PJ and HZ wrote, reviewed and/or revised the manuscript. HZ was also involved in critically revising the manuscript. All authors read and approved the final manuscript.

\section{Ethics approval and consent to participate}

The present study was reviewed and approved by The Second Affiliated Hospital of Kunming Medical University. All animals were raised according to the protocols approved by the Kunming Medical Experimental Animal Care Commission.

\section{Patient consent for publication}

Not applicable.

\section{Competing interests}

The authors declare that they have no competing interests.

\section{References}

1. Torre LA, Bray F, Siegel RL, Ferlay J, Lortet-Tieulent J and Jemal A: Global cancer statistics, 2012. CA Cancer J Clin 65: 87-108, 2015.

2. Center MM, Jemal A, Smith RA and Ward E: Worldwide variations in colorectal cancer. CA Cancer J Clin 59: 366-378, 2009.

3. Cottrell S, Bicknell D, Kaklamanis L and Bodmer WF: Molecular analysis of apc mutations in familial adenomatous polyposis and sporadic colon carcinomas. Lancet 340: 626-630, 1992.

4. Lengauer C, Kinzler KW and Vogelstein B: Genetic instabilities in human cancers. Nature 396: 643-649, 1998.

5. Macfarlane SR, Seatter MJ, Kanke T, Hunter GD and Plevin R Proteinase-activated receptors. Pharmacol Rev 53: 245-282, 2001.

6. Yu G, Jiang P, Xiang Y, Zhang Y, Zhu Z, Zhang C, Lee S, Lee W and Zhang Y: Increased expression of protease-activated receptor 4 and trefoil factor 2 in human colorectal cancer. PLoS One 10: e0122678, 2015.

7. Zhang Y, Yu G, Jiang P, Xiang Y, Li W, Lee W and Zhang Y: Decreased expression of protease-activated receptor 4 in human gastric cancer. Int J Biochem Cell Biol 43: 1277-1283, 2011.

8. Li SM, Jiang P, Xiang Y, Wang WW, Zhu YC, Feng WY, Li SD and Yu GY: Protease-activated receptor (par)1, par2 and par4 expressions in esophageal squamous cell carcinoma. Dongwuxue Yanjiu 35: 420-425, 2014

9. Jiang P, Yu GY, Zhang Y, Xiang Y, Hua HR, Bian L, Wang CY, Lee WH and Zhang Y: Down-regulation of protease-activated receptor 4 in lung adenocarcinoma is associated with a more aggressive phenotype. Asian Pac J Cancer Prev 14: 3793-3798, 2013.

10. Lee S, Jiang P, Wang W, Feng W and Yu G: The decreased expression of protease-activated receptor 4 in esophageal squamous carcinoma. Neoplasma 61: 546-552, 2014.

11. Elste AP and Petersen I: Expression of proteinase-activated receptor 1-4 (par 1-4) in human cancer. J Mol Histol 41: 89-99, 2010.

12. Darmoul D, Gratio V, Devaud H, Lehy T and Laburthe M: Aberrant expression and activation of the thrombin receptor protease-activated receptor- 1 induces cell proliferation and motility in human colon cancer cells. Am J Pathol 162: 1503-1513, 2003.
13. Booden MA, Eckert LB, Der CJ and Trejo J: Persistent signaling by dysregulated thrombin receptor trafficking promotes breast carcinoma cell invasion. Mol Cell Boil 24: 1990-1999, 2004.

14. Kaufmann R, Rahn S, Pollrich K, Hertel J, Dittmar Y, Hommann M, Henklein P, Biskup C, Westermann M, Hollenberg MD and Settmacher U: Thrombin-mediated hepatocellular carcinoma cell migration: Cooperative action via proteinase-activated receptors 1 and 4. J Cell Physiol 211: 699-707, 2007.

15. Kaufmann R, Junker U, Nuske K, Westermann M, Henklein P, Scheele J and Junker K: Par-1- and par-3-type thrombin receptor expression in primary cultures of human renal cell carcinoma cells. Int J Oncol 20: 177-180, 2002.

16. Segal L, Katz LS, Shapira H, Sandbank J, Geras-Raaka E, Gershengorn MC and Oron Y: Par-3 knockdown enhances adhesion rate of panc- 1 cells via increased expression of integrinalphav and e-cadherin. PloS One 9: e93879, 2014.

17. Greenberg DL, Mize GJ and Takayama TK: Protease-activated receptor mediated rhoa signaling and cytoskeletal reorganization in lncap cells. Biochemistry 42: 702-709, 2003.

18. Huang YQ, Li JJ and Karpatkin S: Thrombin inhibits tumor cell growth in association with up-regulation of p21(waf/cip1) and caspases via a p53-independent, stat-1-dependent pathway. J Boil Chem 275: 6462-6468, 2000.

19. Gratio V, Walker F, Lehy T, Laburthe M and Darmoul D: Aberrant expression of proteinase-activated receptor 4 promotes colon cancer cell proliferation through a persistent signaling that involves src and erbb-2 kinase. Int J Cancer 124: 1517-1525, 2009.

20. Yu G, Lee YC, Cheng CJ, Wu CF, Song JH, Gallick GE, Yu-Lee LY, Kuang J and Lin SH: Rsk promotes prostate cancer progression in bone through ing3, ckap2, and ptk6-mediated cell survival. Mol Cancer Res 13: 348-357, 2015.

21. Zhang Y, Yu G, Wang Y, Xiang Y, Gao Q, Jiang P, Zhang J, Lee $\mathrm{W}$ and Zhang Y: Activation of protease-activated receptor (par) 1 by frog trefoil factor (tff) 2 and par4 by human tff2. Cell Mol Life Sci 68: 3771-3780, 2011.

22. Sambrano GR, Weiss EJ, Zheng YW, Huang W and Coughlin SR: Role of thrombin signalling in platelets in haemostasis and thrombosis. Nature 413: 74-78, 2001.

23. Kataoka H, Hamilton JR, McKemy DD, Camerer E, Zheng YW, Cheng A, Griffin C and Coughlin SR: Protease-activated receptors 1 and 4 mediate thrombin signaling in endothelial cells. Blood 102: 3224-3231, 2003.

24. Bretschneider E, Kaufmann R, Braun M, Nowak G, Glusa E and Schror K: Evidence for functionally active protease-activated receptor-4 (par-4) in human vascular smooth muscle cells. Br J Pharmacol 132: 1441-1446, 2001.

25. Mußbach F, Henklein P, Westermann M, Settmacher U, Bohmer FD and Kaufmann R: Proteinase-activated receptor 1and 4-promoted migration of hep3b hepatocellular carcinoma cells depends on ros formation and rtk transactivation. J Cancer Res Clin Oncol 141: 813-825, 2015.

26. Sedda S, Marafini I, Caruso R, Pallone F and Monteleone G: Proteinase activated-receptors-associated signaling in the control of gastric cancer. World J Gastroenterol 20: 11977-11984, 2014.

27. Smith TH, Coronel LJ, Li JG, Dores MR, Nieman MT and Trejo J: Protease-activated receptor- 4 signaling and trafficking is regulated by the clathrin adaptor protein complex-2 independent of $\beta$-arrestins. J Boil Chem 291: 18453-18464, 2016.

This work is licensed under a Creative Commons Attribution-NonCommercial-NoDerivatives 4.0 International (CC BY-NC-ND 4.0) License. 